DE

M E D I C I N A

T R O P I C A L

$\mathrm{DE}$

SÃO PAULO

JOURNAL OF THE SÃO PAULO INSTITUTE OF TROPICAL MEDICINE

${ }^{1}$ Fundação Oswaldo Cruz Rondônia, Porto Velho, Rondônia, Brazil

¿Universidade Federal de Rondônia, Programa de Pós-Graduação em Biologia Experimental, Porto Velho, Rondônia, Brazil

${ }^{3}$ Centro de Pesquisa em Medicina Tropical, Porto Velho, Rondônia, Brazil

${ }^{4}$ Instituto de Biologia Molecular do Paraná, Curitiba, Paraná, Brazil

5Universidade Federal do Paraná, Departamento de Engenharia de Bioprocessos e Biotecnologia, Curitiba, Paraná, Brazil

${ }^{6}$ Instituto Carlos Chagas, Curitiba, Paraná, Brazil

Correspondence to: Rita de Cássia Pontello Rampazzo Instituto de Biologia Molecular do Paraná, Rua Professor Algacyr Munhoz Mader, 3775, CEP 81350-010, Curitiba, PR, Brazil Tel: +554133163260

E-mail: rcprampazzo@gmail.com, rirampazzo@ibmp.org.br

Received: 27 March 2019

Accepted: 5 July 2019

\section{Epidemiological profile of Zika, Dengue and Chikungunya virus infections identified by medical and molecular evaluations in Rondonia, Brazil}

\author{
Deusilene Souza Vieira ${ }^{1,2,3}$, Miriam Ribas Zambenedetti ${ }^{4}$, Luciana Requião ${ }^{4}$, \\ Ivo Alberto Borghetti ${ }^{4,5}$, Luciano Kleber de Souza Luna ${ }^{4}$, Alcione de \\ Oliveira dos Santos ${ }^{1,3}$, Roger Lafontaine Mesquita Taborda ${ }^{3}$, Dhelio Batista \\ Pereira $^{3}$, Marco Aurélio Krieger ${ }^{4,6}$, Juan Miguel Villalobos Salcedo ${ }^{1,3}$, Rita de \\ Cássia Pontello Rampazzo 4
}

\section{ABSTRACT}

Several arboviruses have emerged and/or re-emerged in North, Central and SouthAmerican countries. Viruses from some regions of Africa and Asia, such as the Zika and Chikungunya virus have been introduced in new continents causing major public health problems. The aim of this study was to investigate the presence of RNA from Zika, Dengue and Chikungunya viruses in symptomatic patients from Rondonia, where the epidemiological profile is still little known, by one-step real-time RT-PCR. The main clinical signs and symtoms were fever $(51.2 \%)$, headache $(78 \%)$, chills $(6.1 \%)$, pruritus $(12.2 \%)$, exanthema $(20.1 \%)$, arthralgia $(35.3 \%)$, myalgia $(26.8 \%)$ and retro-orbital pain $(19.5 \%)$. Serum from 164 symptomatic patients were collected and tested for RNA of Zika, Dengue types 1 to 4 and Chikungunya viruses, in addition to antibodies against Dengue NS1 antigen. Direct microscopy for Malaria was also performed. Only ZIKV RNA was detected in $4.3 \%$ of the patients, and in the remaining $95.7 \%$ of the patients RNA for Zika, Dengue and Chikungunya viruses were not detected. This finding is intriguing as the region has been endemic for Dengue for a long time and more recently for Chikungunya virus as well. The results indicated that medical and molecular parameters obtained were suitable to describe the first report of symptomatic Zika infections in this region. Furthermore, the low rate of detection, compared to clinical signs and symptoms as the solely diagnosis criteria, suggests that molecular assays for detection of viruses or other pathogens that cause similar symptoms should be used and the corresponding diseases could be included in the compulsory notification list.

KEYWORDS: Zika. Chikungunya. Dengue. One-step real-time RT-PCR. Diagnostic. Arboviruses.

\section{INTRODUCTION}

Climate conditions together with large populations of mosquitoes from the genera Aedes sp. and Anopheles sp. in the Western Brazilian Amazon lead to the perfect environment for the maintenance of arboviruses and protozoa transmission cycles. Outbreaks of Dengue virus (DENV), Yellow Fever virus (YFV), Oropouche virus (OROV), Mayaro virus (MAYV) and the Plasmodium sp. protozoa parasites have already been reported in this region ${ }^{1-4}$.

The Zika virus (ZIKV) was first isolated in 1947 from a monkey in Uganda and sporadic infections were subsequently identified in humans. In 2007, the virus was 
found to be widespread among patients presenting with flu-like symptomatology during an outbreak in the Yap Island of the Federated States of Micronesia. The subsequent increased incidence of patients with microcephaly or GuillainBarré Syndrome in Brazil has been intensively investigated and the resulting data have established an association between ZIKV infections and neurological disorders ${ }^{5-7}$.

Infection with the DENV normally leads to fever and flu-like signs and symptoms, but can also be fatal in some cases $^{8,9}$. Based on the epidemiological investigations reported to the World Health Organization, an estimated 50-100 million cases occur annually in more than 100 endemic countries, which indicates that half of the world's population is at risk ${ }^{9}$.

Chikungunya virus (CHIKV) infection was first reported in 1952 in Southern Tanzania. Chikungunya fever is characterized by joint pains and high fever. The virus has recently arrived in Brazil (2014), and from the first report to the second half of 2017, approximately 300,000 cases have been reported, and $40 \%$ of Brazilian municipalities had confirmed cases ${ }^{10,11}$.

Vector-borne diseases have been a major challenge for public health, with mosquitoes being the main vectors of hundreds of microorganisms around the world ${ }^{12}$. The ecological and environmental conditions of the Amazon region favor the abundant presence of several species, including those that are potential vectors of human pathogens ${ }^{13}$.

Arboviruses have been a significant and constant threat due to the ease of adaptation of these viruses to new environments and their capacity to infect both vertebrates and invertebrate hosts. However, the anthropogenic environmental factors, such as disorganized urbanization, population displacement and the precariousness of basic sanitation, have favored the transmission and spread of these viruse ${ }^{14}$.

The aim of this study was to investigate the presence of main arboviruses spreading in Brazil (ZIKV, DENV and CHIKV) in patients with symptoms suggestive of arboviral disease, who lived in Rondonia during the first half of 2017. In summary, to date, this study provided the first epidemiological data on patients in this region, in whom RNA from ZIKV were identified. It is suggested that different protocols for the diagnosis of arbovirus infections should be combined in order to develop an improved algorithm to identify specific pathogens.

\section{MATERIAL AND METHODS}

\section{Study location}

The study was developed at the Ambulatory of Malaria
Outpatient Center for Tropical Medicine (CEPEM), located at BR 364, Km 3.5 in the city of Porto Velho, an agency linked to the State Department of Health (SESAU). The outpatient clinic is a reference center in malaria, attending hundreds of people with characteristic symptoms of the infection.

\section{Samples}

This study was approved by the local Research Ethics Committee (1.474.102CEP/2016/CEPEM). Whole blood samples were collected during the first half of 2017. For this study, serum samples from symptomatic patients were used and there was no laboratory evidence of the presence of Plasmodium sp. according to the direct microscopy investigation. In the first moment, a medical consultation was carried out to investigate the clinical data and identify the symptoms in these patients. Individuals over 12 years of age and both sexes, whose symptoms had a clinical course of up to five days, were included. The participant must also been agreed to receive periodic telephone calls and other electronic media contacts. Exclusion criteria were patients who do not have samples for laboratory and immunological characterization, indigenous patients and patients from other vulnerable groups.

\section{RNA extraction}

Viral RNA was extracted from $140 \mu \mathrm{L}$ of serum samples by the QIAamp ${ }^{\circledast}$ Viral RNA Mini Kit (QIAGEN, Germany), according to the manufacturer's instructions. RNA was eluted in $60 \mu \mathrm{L}$ of the AVE buffer.

\section{Real-time RT-PCR}

The RNA samples were tested for the presence of ZIKV; DENV serotypes 1, 2, 3, and 4; and CHIKV by a one-step real-time RT-PCR Kit (Biomol ZDC, Instituto de Biologia Molecular do Parana, Brazil), following the manufacturer's instructions. Reactions were performed in an Applied Biosystems 7500 Real-Time PCR apparatus, as follows: $51{ }^{\circ} \mathrm{C}$ for $30 \mathrm{~min}, 95^{\circ} \mathrm{C}$ for $15 \mathrm{~min}, 40$ cycles of $95^{\circ} \mathrm{C}$ for $15 \mathrm{~s}$, and $60^{\circ} \mathrm{C}$ for $1 \mathrm{~min}$. Reactions were considered positive when the threshold cycle was $\leq 36$ for the virus analyzed and $\leq 28$ for the internal control.

\section{DENV NS1 antigen testing}

Serum samples were tested using the Platelia DENV NS1 AG (BIO-RAD, USA), an enzyme immunoassay for qualitative or semiquantitative detection of the NS1 antigen in human serum or plasma. 


\section{Direct microscopy for malaria}

Thick blood smears were stained with Giemsa which is the gold standard method for malaria diagnosis. Each blood smear was examined for the presence of Plasmodium by experienced technicians.

\section{Statistical analysis}

Data were entered into a database in Excel. The prevalences were analyzed with the WinEpi on-line package (Universidad de Zaragoza, Spain) and were presented as absolute values, with $95 \%$ confidence intervals (95\% CIs). All statistical analyses were performed using the Prism ${ }^{\circledR}$ 7.01 software for Windows ${ }^{\mathrm{TM}}$ (GraphPad Software, San Diego, CA). In all cases, $\mathrm{p}$ values $<0.05$ were considered statistically significant.

\section{RESULTS}

A total of 164 samples were collected during the first half of 2017, from patients whose symptoms were mostly self-declared. During the anamnesis, the main relevant signs and symptoms reported by patients were: headache 78/164 (78\%), fever 84/164 (51.2\%), arthralgia 58/164 (35.3\%), myalgia 44/164 (26.8\%), exanthema 33/164 (20.1\%), retroorbital pain 32/164 (19.5\%), pruritus 20/164 (12.2\%), chills 10/164 (6.1\%) and conjunctivitis 7/164 (4.3\%). Correlations between signs and symptoms of ZIKV-positive or negative patients are shown in Table 1.

Among the signs and symptoms reported by patients with viremia, only conjunctivitis had a statistical relevance $(\mathrm{OR}=12.16,95 \% \mathrm{CI}, 1.88-78.63, \mathrm{p}=0.03)$, with a positive correlation between conjunctivitis and positivity for ZIKV, although only two cases had conjunctivitis.
RNA from the serum samples of 164 patients were tested by real-time RT-PCR. Amplification results revealed that 7 (4.3\%) samples were positive only for ZIKV, and 157 were negative for all DENV serotypes, CHIKV and ZIKV viruses. Negative real-time RT-PCR results were validated by the internal control (Figure 1). All RNA samples amplified the internal control sequence (an endogenous human transcript), ensuring that these samples were not degraded and that the negative results were true negatives. The serological test for NS1 DENV antigen was negative for all serum samples. The microscopic evaluation of 164 peripheral blood smears was negative for malaria infection. In addition, the specificity of the real-time RT-PCR assay (Kit Biomol ZDC) for ZIKV, DENV and CHIKV was assessed by testing samples known to be positive for Plasmodium falciparum; MAYV, YFV and OROV; as well as ZIKV, DENV1, DENV2, DENV3, DENV4 and CHIKV. Data showed specific amplifications only, confirming the absence of cross-reactivity with the other evaluated pathogens. Assays were validated by the positive control supplied by the manufacturer (Figure 2).

\section{DISCUSSION}

Infections caused by arboviruses and Plasmodium sp. occur in the same geographical regions due to the association between the climate and the presence of the vectors Aedes sp. and Anopheles sp., respectively. Patients with these infections present similar signs and symptoms, including chills, fever and headache, which may be mild and difficult to recognize ${ }^{1,12-14}$. In this study, considering the signs and symptoms reported by patients with viremia, only conjunctivitis was more common in ZIKV-positive cases, with $28.6 \%$ of patients with ZIKV infection presenting the symptom, corroborating results described in the literature ${ }^{15,16}$. Although conjunctivitis is reported in cases

Table 1 - Signs and symptoms reported by patients.

\begin{tabular}{|c|c|c|c|c|c|c|}
\hline \multirow{2}{*}{ Signs and symptoms } & \multicolumn{2}{|c|}{ Positive ZIKV } & \multicolumn{2}{|c|}{ Negative ZIKV } & \multirow{2}{*}{$p$ value } & \multirow{2}{*}{ OR $(95 \% \mathrm{Cl})$} \\
\hline & $n=7$ & $(\%)$ & $\mathrm{n}=157$ & $(\%)$ & & \\
\hline Fever & 5 & 71.4 & 79 & 50.3 & 0.274 & $2.47(0.46-13.11)$ \\
\hline Arthralgia & 4 & 57.1 & 54 & 34.4 & 0.245 & $2.54(0.55-11.78)$ \\
\hline Myalgia & 3 & 42.9 & 41 & 26.1 & 0.387 & $2.12(0.45-9.89)$ \\
\hline Exanthema & 3 & 42.9 & 30 & 19.1 & 0.146 & $3.17(0.67-14.95)$ \\
\hline Itching & 2 & 28.6 & 18 & 11.5 & 0.204 & $3.09(0.56-17.12)$ \\
\hline Retro-Orbital Pain & 3 & 42.9 & 29 & 18.5 & 0.135 & $3.31(0.70-15.61)$ \\
\hline Headache & 3 & 42.9 & 75 & 47.8 & 1.000 & $0.82(0.18-3.79)$ \\
\hline Chills & 0 & 0.0 & 10 & 6.4 & 1.000 & $0.94(0.05-17.56)$ \\
\hline Conjunctivitis & 2 & 28.6 & 5 & 3.2 & 0.030 & $12.16(1.88-78.63)$ \\
\hline
\end{tabular}

OR - Odds ratio; $\mathrm{Cl}$ - Confidence interval. 


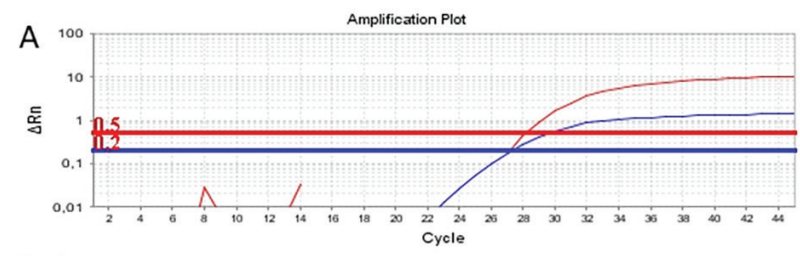

CONTROL Zika

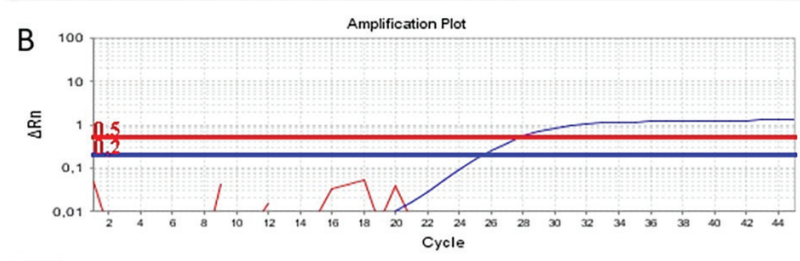

CONTROL ZIKa

Figure 1 - Amplification profile depicting the threshold cycles (Cts) of positive patients and negative ones: A) Red line: positive ZIKV patient; B) Blue line: a negative patient to ZIKV, showing only the amplification of the internal control.

of ZIKV, it is not pathognomonic of this infection, as it has already been described in cases of DENV infection ${ }^{16}$, and has been observed in this study in $3.2 \%$ of ZIKV, DENV and CHIKV-negative cases, in infections probably caused by other pathogens.

One hundred and sixty-four patients presenting with symptomatology suggestive of arboviral infections were evaluated by medical, molecular and microscopic methods and, in this study, the first cases of symptomatic ZIKV infections were identified in Rondonia State, by a one-step real-time RT-PCR assay, after a worldwide explosive outbreak of the virus in 2015.

The Epidemiological Bulletin from the Secretariat of Health Surveillance, Ministry of Health ${ }^{17}$, reported that the DENV prevalence in Rondonia State during 2017, based only on clinical signs and symptoms, was of 1,807 cases. For ZIKV and CHIKV, the numbers were 101 and 163, respectively. However, in this study, the detection rate of viral ZIKV RNA was $4.3 \%$ in the same region. Thus, our results suggest that the notification based only on epidemiological criteria and clinical symptomatology is unsuitable as diagnoses are not always confirmed by laboratory tests. Our cohort was composed of only symptomatic patients, and the number of ZIKV confirmed cases was low. To circumvent this problem, we suggest the use of molecular diagnostic protocols to assess the etiologic agents of arboviruses and other pathogens circulating in Brazil.

The nucleic acid assay ZDC BioMol kit (IBMP) used to detect RNA from ZIKV, DENV and CHIKV was effective in a previous epidemiological study, using different types of biological specimens such as urine and saliva samples, demonstrating that is an important tool for identification and differentiation of these arboviruses ${ }^{18}$.

Several surveillance strategies have been carried out for ZIKV, DENV and CHIKV infections in some localities in Northern Brazil. However, no screening study for ZIKV had been previously performed in Rondonia State. Currently, there are ongoing surveys on DENV and more recently on CHIKV fever (e.g., first description of CHIKV in Amapa State) $)^{19}$.
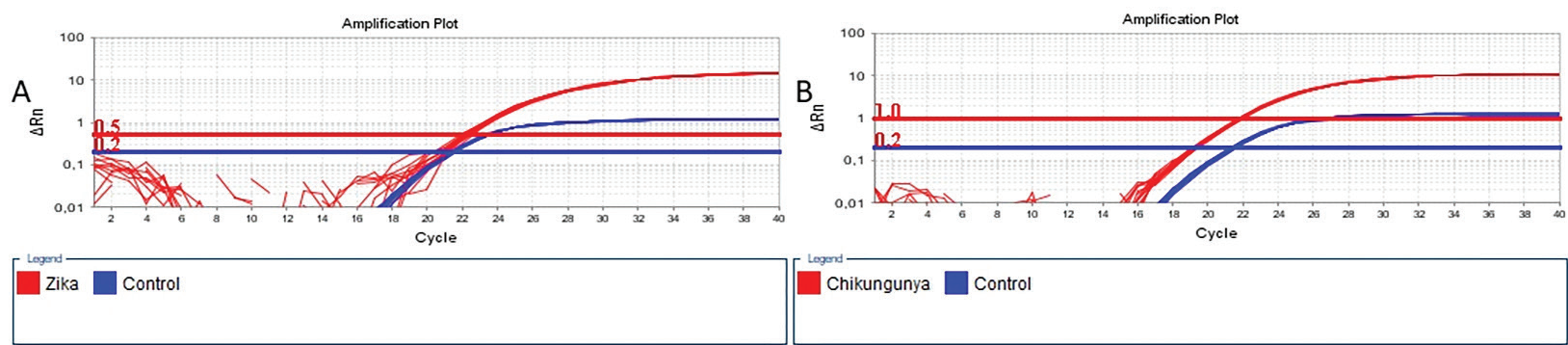

Chikungunya $\square$ Control
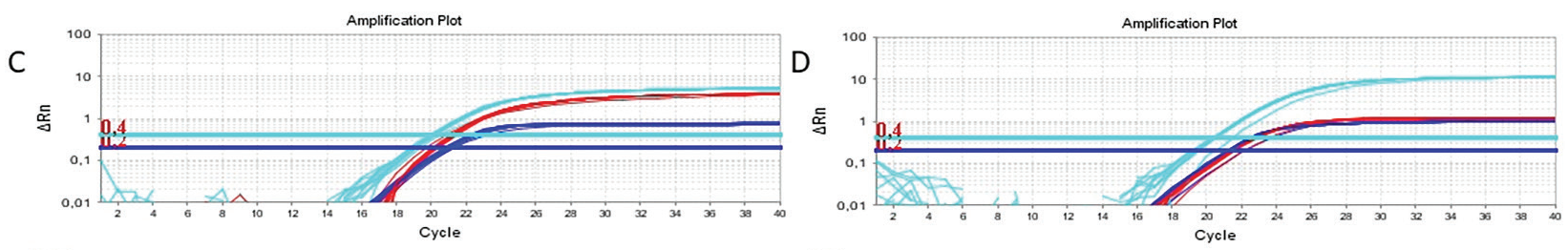

Dengue1 Dengue4 $\square$ Control

Dengue3 $\square$ Control $\square$ Dengue2

Figure 2 - Amplification profiles depicting the Cts (on the abscissa axis) of positive samples for ZIKV, CHIKV, and DENV and the controls supplied by the Kit Biomol ZDC: A) Zika; B) Chikungunya; C) Dengue 1 and Dengue 4; D) Dengue 2 and Dengue 3 . The amplification profiles of the studied viruses are represented in red or turquoise, while the controls are always represented in blue. The emitted fluorescence threshold (on the ordinate axis) for Zika is 0.5 ; for Dengue 1 and the Control is 0.2 ; for Dengue 2 is 0.5 and for Dengue 3 and 4 is 0.4 and for Chikungunya is 1. 
The time lapse from the first molecular detection of ZIKV in Rondonia State to the first reported detection of autochthonous ZIKV in Brazil ${ }^{20}$ suggests that this virus is in the process of spreading in this region, as previously described for DENV and CHIKV.

The small number of studies, together with the difficulty of identifying ZIKV, may be associated with geographic characteristics of the region. In addition, the co-circulation of other arboviruses, such as DENV, CHIKV, MAYV and OROV, which have overlapping symptoms to ZIKV infection (low fever, pruritus and cutaneous rash), may pose an additional challenge to understand the epidemiology of ZIKV infection in Rondonia State. Furthermore, the literature describes that the percentage of Zika asymptomatic or mild symptomatic cases is approximately $80 \%$ and this factor could be associated with our symptomatic cohort $\mathrm{t}^{21,22}$.

It is worth noting that DENV was not detected in this study, even by serological and molecular methods and considering that the region is endemic to this virus ${ }^{23}$.

Our new findings are of paramount importance to public health authorities, as ZIKV infections, as observed mainly in the Northeast region, has been responsible for the occurrence of complicated neurological syndromes in Brazil ${ }^{24}$. Due to the dispersion of specific ZIKV vectors throughout the country, in addition to the other major arboviruses, molecular screening should be considered as an important tool for monitoring patients with acute febrile diseases. Finally, the low rate of detection of ZIKV-infected individuals (7/164) and the absence of positive cases for DENV, CHIKV and Plasmodium sp. infections suggest that other pathogens have been circulating in this area. Because several outbreaks of arboviruses infections in the Rondonia have been reported, especially of $\mathrm{DENV}^{23}$, medical and molecular investigations for new arboviruses that are spreading throughout this region are needed. The list of new viruses should include the West Nile virus (WNV), MAYV, YFV and OROV.

\section{CONCLUSION}

To the best of our knowledge, this study described the first molecular evidence of human symptomatic ZIKV infections in Rondonia, a region considered highly endemic for arboviruses. As the signs and symptoms presented by the investigated population are similar to those of other arboviruses, an accurate laboratory scenario for the molecular screening of the main circulating arboviruses in our region is of prime importance.

\section{CONFLICT OF INTERESTS}

The authors declare no potential conflict of interest.

\section{ACKNOWLEDGMENTS}

The authors would like to thank the Instituto Nacional de Epidemiologia da Amazônia Ocidental (INCT-EpiAmO), Fundação Rondônia de Amparo ao Desenvolvimento das Ações Científicas e Tecnológicas e à Pesquisa do Estado de Rondônia (FAPERO), Decit/ SCTIE/MS, CNPq, SESAU-RO and the Instituto de Biologia Molecular do Paraná (IBMP) for their assistance in conducting the study.

\section{REFERENCES}

1. Mourão MP, Bastos MS, Figueiredo RM, Gimaque JB, Alves VC, Saraiva MG, et al. Arboviral diseases in the Western Brazilian Amazon : a perspective and analysis from a tertiary health \& research center in Manaus, State of Amazonas. Rev Soc Bras Med Trop. 2015;48 Suppl 1:20-6.

2. Vieira DS, Honda ER, Pereira SS, Bifano GS, Tada MS, Batista WC. Characterization of dengue virus serotype 1 in epidemics in Porto Velho, Rondônia, in 2001-2003. Rev Soc Bras Med Trop. 2007;40:268-71.

3. World Health Organization. World malaria report 2018. Geneva: WHO; 2018. [cited 2019 Mar 27]. Available from: https://www.who.int/malaria/publications/world-malariareport-2018/en/

4. Vieira DS, Bifano GS, Honda ER, Tada MS, Batista WC. Isolation and identification of Dengue virus serotype 3 in Rondonia, Brazil. Virus Rev Res. 2008;13:1-16.

5. Plourde AR, Bloch EM. A literature review of Zika virus. Emerg Infect Dis. 2016;22:1185-92.

6. Brasil P, Pereira Jr JP, Moreira ME, Nogueira RM, Damasceno $\mathrm{L}$, Wakimoto M, et al. Zika virus infection in pregnant women in Rio de Janeiro. N Engl J Med. 2016;375:2321-34.

7. Araujo LM, Ferreira ML, Nascimento OJ. Guillain-Barré syndrome associated with the Zika virus outbreak in Brazil. Arq Neuropsiquiatr. 2016;74:253-5.

8. Bhatt S, Gething PW, Brady OJ, Messina JP, Farlow AW, Moyes $\mathrm{CL}$, et al. The global distribution and burden of dengue. Nature. 2013;496:504-7.

9. World Health Organization. Dengue control: epidemiology. [cited 2019 Mar 27]. Available from: https://www.who.int/ denguecontrol/epidemiology/en

10. World Health Organization. Chikungunya. [cited 2019 Mar 27]. Available from: https://www.who.int/en/news-room/factsheets/detail/chikungunya

11. Cunha RV, Trinta KS. Chikungunya virus : clinical aspects and treatment - A review. Mem Inst Oswaldo Cruz. 2017;112:52331.

12. Cheng G, Liu Y, Wang P, Xiao X. Mosquito defense strategies against viral infection. Trends Parasitol. 2016;32:177-86. 
13. Laporta GZ, Ribeiro MC, Ramos DG, Sallum MA. Spatial distribution of arboviral mosquito vectors (Diptera, Culicidae) in Vale do Ribeira in the South-eastern Brazilian Atlantic Forest. Cad Saude Publica. 2012;28:229-38.

14. Donalisio MR, Freitas AR, Von Zuben AP. Arboviruses emerging in Brazil: challenges for clinic and implications for public health. Rev Saude Publica. 2017;51:30.

15. Heukelbach J, Alencar CH, Kelvin AA, Oliveira WK, Cavalcanti LP. Zika virus outbreak in Brazil. J Infect Dev Ctries. 2016;10:116-20.

16. Colombo TE, Estofolete CF, Reis AF, Silva NS, Aguiar ML, Cabrera EM, et al. Clinical, laboratory and virological data from suspected ZIKV patients in an endemic arbovirus area. J Clin Virol. 2017;96:20-5.

17. Brasil. Ministério da Saúde. Secretaria de Vigilância em Saúde. Monitoramento dos casos de dengue, febre de chikungunya e doença aguda pelo vírus Zika até a Semana Epidemiológica 30 de 2018. Bol Epidemiol. 2018;49(34):1-14.

18. Bonaldo MC, Ribeiro IP, Lima NS, Santos AA, Menezes LS, Cruz $\mathrm{SO}$, et al. Isolation of infective Zika virus from urine and saliva of patients in Brazil. PLoS Negl Trop Dis. 2016;10:e004816.
19. Honório NA, Câmara DC, Calvet GA, Brasil P. Chikungunya: an arbovirus infection in the process of establishment and expansion in Brazil. Cad Saude Publica. 2015;31:906-8.

20. Zanluca C, Melo VC, Mosimann AL, Santos GI, Santos CN, Luz K. First report of autochthonous transmission of Zika virus in Brazil. Mem Inst Oswaldo Cruz. 2015;110:569-72.

21. Proenca-Modena JL, Milanez GP, Costa ML, Judice CC, Maranhão Costa FT. Zika virus: lessons learned in Brazil. Microbes Infect. 2018;20:661-9.

22. Haby MM, Pinart M, Elias V, Reveiz L. Prevalence of asymptomatic Zika virus infection: a systematic review. Bull World Health Organ. 2018;96;402-13D.

23. Salles TS, Sá-Guimarães TE, Alvarenga ES, Guimarães-Ribeiro V, Meneses MD, Castro-Salles PF, et al. History, epidemiology and diagnostics of dengue in the American and Brazilian contexts: a review. Parasit Vectors. 2018;11:264.

24. World Health Organization. Emergencies: archived Zika virus classification tables. [cited 2019 Mar 27]. Available from: https://www.who.int/emergencies/zika-virus/classificationtables/en/ 